\title{
THE INFLUENCE OF THE VELOCITY DISPERSION ON THE VELOCITY-DENSITY RELATION IN THE DISK MODEL OF THE GALAXY
}

\author{
Szymon Sikora $^{\mathrm{a}}$, ŁukAsz BrateK ${ }^{\mathrm{b}, \mathrm{c}}$, JoAnna JaŁocha ${ }^{\mathrm{c}}$ \\ MAREK KUTSChERA ${ }^{\mathrm{d}}$ \\ ${ }^{a}$ Astronomical Observatory, Jagiellonian University \\ Orla 171, 30-244 Kraków, Poland \\ ${ }^{b}$ H. Niewodniczański Institute of Nuclear Physics, Polish Academy of Sciences \\ Radzikowskiego 152, 31-342 Kraków, Poland \\ 'Institute of Physics, Cracow University of Technology \\ Warszawska 24, 30-084 Kraków, Poland \\ ${ }^{\mathrm{d}}$ M. Smoluchowski Institute of Physics, Jagiellonian University \\ Łojasiewicza 11, 30-348 Kraków, Poland
}

(Received October 9, 2017; accepted November 17, 2017)

Taking the velocity dispersion term into account in the Jeans equation describing the disk model, counterintuitively allows to reduce the local mass-to-light ratio at the outskirts of flattened galaxies, and stop the unbound growth of mass function. This is possible thanks to a more intertwined relation between the gravitational potential and the rotation curve than for spheroidal systems. This effect is illustrated on the example of galaxy UGC 6446 by finding iteratively the column mass density from the rotation curve supplemented with an isotropic velocity dispersion profile in the disk plane. Along with galactic magnetic fields, this effect would allow to reduce the local mass-to-light ratio at the galactic outskirts.

DOI:10.5506/APhysPolB.48.2071

\section{Introduction}

Modeling of rotation curves presupposes that galaxies are in virialized steady state rotation. To simplify things, one usually assumes: axial symmetry, a number of fixed shape mass subcomponents, constrained density-luminosity (most often a constant mass-to-light ratio), and constrained density-dispersion profiles in the disk component; see Sofue [1] for a review. The simplifications reduce a complicated dynamical model of a galaxy to only several analytical formulas with free parameters. For example, based on 
the van der Kruit and Searle [2] result, Leroy et al. [3] relate the stellar vertical velocity dispersion $\sigma_{*, z}$ and the stellar disk mass density $\Sigma_{*}$ by assuming the stellar disk to be isothermal in the vertical direction, with exponential radial and vertical profiles and a width-scale independent of the radial variable. Similar constraints and constant mass-to-light ratio are adopted by Watson et al. [4] and Martinsson et al. [5] ${ }^{1}$. The obtained gravitating mass is higher than expected based on the brightness curve [7]. The difference between the dynamical mass and the observed distribution of stars and gas is too high to be attributable entirely to nonluminous baryonic component (brown dwarfs, molecular gas). The missing mass manifests itself especially in the outer galactic regions where the mass-to-light ratio is significantly higher than that characteristic of stellar populations $[8]^{2}$.

In this paper, we study the effect of the velocity dispersion term in Jeans equations on the predicted mass distribution and on the resulting local massto-light ratio behavior at the outskirts of flattened galaxies. In calculating the local mass-to-light ratio, we consider the total gravitating mass after subtraction of the gas contribution. We assume as a working hypothesis that there is no significant spheroidal mass component dominating other mass components at larger distances. We abandon at the same time the assumption of constant mass-to-light ratio. Of interest in this paper is the gravitational field at larger distances only, where the shape of the central regions, whether spherical or disk-like, is not much important as their contribution to the gravitational potential in the far region is dominated by their monopole term. Then, the use of the thin disk model is better justified than the use of a spherical model. With nonzero dispersion term in this situation, as we show in this paper, it is possible to significantly reduce the local massto-light ratio at the galactic outskirts without any noticeable change in the mass function. The reduction effect is best seen at the galactic edge, where the density is small, thus perturbations of the density relatively more pronounced, by which these regions become interesting due to the sensitivity of the rotation curve in disk model to local gradients in the mass distribution.

Under such circumstances, particularly interesting are galaxies for which the disk model with vanishing velocity dispersion would lead to divergent local mass-to-light ratio, suggesting significant amounts of nonluminous matter. One perfect candidate to be studied is the UGC 6446 galaxy. As suggested by Jałocha et al. [11], this galaxy might be flattened rather than dominated by a spherical component. Some features of this galaxy suggest large amounts of nonbaryonic dark matter, and other that the dark matter

\footnotetext{
${ }^{1}$ For a detailed discussion of the stellar mass-to-light ratio estimation from galactic spectra, see [6] and the references therein.

${ }^{2}$ We refer to Schaye [9] and van der Kruit [10] for complete picture on the star formation threshold and the gaseous disk structure at the galaxy edge.
} 
mass may be not that high. For example, the local mass-to-light ratio calculated after subtraction of the gas contribution, grows rapidly at the edge of the stellar disk. It is then important to consider other factors, which the high mass-to-light ratio is sensitive to in the boundary region, as they could occur to reduce this ratio. The velocity dispersion is such a factor, influencing the rotation-density relation and, therefore, the resulting mass-to-light ratio behavior.

The dynamical role of the velocity dispersion can be intuitively understood in terms of a reduced dispersion tensor described by a single scalar pressure. The sign of the corresponding term in Jeans equations is opposite to that of the gravitational term in regions where the density and pressure decreases with the distance. This implies an increase of the gravitational force for a pressure supported galaxy model with a given rotation curve. In particular, approximately spherical such a system must be more massive than that with the same rotation curve and neglected pressure. This is the case also for a pressure supported gaseous disk in the potential of a dominating, nearly spherical mass component $[12]^{3}$. However, the increase of mass characteristic of nearly spherical systems does not generally hold for flattened systems of which rotation curves are strongly modified by local gradients in the density, and these variations are crucial for the disk model, unlike for spherical models sensitive only to the amount of mass encircled by a given orbit. The dispersion term introduces further structural modifications in the rotation law and is also dependent on the required density, which is a functional of the rotation curve (note, that the starting quantity from which the mass density is inferred is the rotation curve only). In consequence of this "backreaction", the relation between the circular rotation and the density profile becomes very complicated in the disk model. The effect of the interplay between the dispersion and the gravitational term for flattened systems can be such that the mass density is locally reduced while leaving the rotation curve unaffected. The effect will be important in regions where the relative variations in the density are large, that is, in the disk edge neighborhood where the density is already low. Therefore, the local massto-light ratio of flattened systems can be strongly modified or reduced at the disk edge without significant increase in the amount of mass.

The dispersion term in the disk model gives a tool for strongly modifying the local mass-to-light ratio predicted by models neglecting the velocity dispersion. In this context, it is important to note that finding solutions of the Jeans equations for disk-like mass distributions requires a freely deformable density profile, otherwise the sensitivity of the disk model to local gradients

${ }^{3}$ In this article, it is assumed that the gas pressure is mainly due to turbulent motions and defined as $p=\rho \sigma^{2}$ (where $\sigma$ describes the radial velocity dispersion and $\rho$ is the density), while the thermal pressure is neglected as less important. 
in the density could not be fully used. In principle, this excludes the popular method of rotation curves fitting that makes use of assumed shape density profiles for various mass components. The method which could be effectively used in this situation is to find solutions by iterations.

The disk model neglecting the dispersion velocity ('cold' disk) was originally proposed by Toomre [13] and developed further by Nordsieck [14]. The rotation curve and mass density in this model are convolutions of each other [15]. Unlike for spheroidal models for which the circular velocity is determined by the amount of encircled mass, in the disk model, the circular velocity is strongly dependent on the radial gradients in the mass density and also on masses distributed outside the circle of a given orbit. For example, pronounced local declines in the density reflect in largely increased velocity followed by correspondingly steep decrease. This can be observed for truncated disks [16]. Similarly, an increase in the density results in correspondingly large local decrease in the velocity [17] — an effect impossible for spherical systems. For this reason, one should refrain from making a priori assumptions about the distribution of masses in various mass components in models where the gravitation of disk component is important. In such situations, more appropriate than parametric models are relaxation methods that converge to a self-consistent model with the minimum number of assumptions. Such a model would allow to describe more general situations and provide a tool for testing the assumptions made by parametric models.

In the disk model, the mass density is not uniquely determined by the observed fragment of rotation curve known out to the so-called cutoff radius, e.g. Sackett [18] and Bratek et al. [17]. This drawback can be sometimes overcome by using additional data about the mass density and by finding iteratively a global rotation curve consistent with these data [19]. In this method, the mass density profile is not a priori assumed. On comparing the density and brightness profiles, it is then possible to predict the local mass-to-light ratio. In Sect. 2, we generalize the iterative method to the case with the velocity tensor isotropic in the disk plane and varying with the radius.

\section{Determining the mass distribution in the disk model by iterations}

We use iteration methods to find the column mass density from the rotation curve and other available data in galaxy models involving disks representing flattened mass subcomponent. The iteration method can be used if, for some reasons, one chooses to give up the less exact method of using fixed-shape density profiles with free parameters. In the disk model neglecting the velocity dispersion term, the mass density calculated for the cut rotation curve with the help of the forward (velocity-to-density) integral, 
would not lead to the original rotation curve after substituting the density to the backward (density-to-velocity) integral. Therefore, iterations are necessary. The other advantage of the iterations is that the final density can be controlled by the measured density of hydrogen, especially if the latter extends further than the rotation curve. In a disk model with the dispersion term included, things complicate further, because the dispersion term also involves the unknown density. Again, one can use iterations and find a self-consistent model of mass density that exactly accounts for the measured rotation curve. An example of iteration method is described in [19]. Below, we extend it to the disk model with the velocity dispersion term included.

In the Jeans theory framework, we consider a stationary axi-symmetric and infinitesimally thin disk in the plane $z=0$ with polar coordinates $(R, \phi)$. The disk surface mass density $\Sigma$, the planar velocity components, and the gravitational potential $\Psi$ are related through the Poisson equation, the continuity equation and the second moment Jeans equations. We split the total mass density $\Sigma(R)$ into the stellar density $\Sigma_{*}(R)$ and the gas density $\Sigma_{\text {gas }}(R)$

$$
\Sigma(R)=\Sigma_{*}(R)+\Sigma_{\text {gas }}(R) .
$$

\subsection{The vanishing velocity dispersion}

We begin with a simpler case when the dispersion tensor is zero. In the approximation of circular orbits, $\Sigma(R)$ and the circular velocity $v(R)$ are related through integral convolutions [15] that for a thin disk can be recast to a particularly simple explicit form [20]

$$
\begin{aligned}
v^{2}(R) & =\int_{0}^{\infty} w(x) \mu(R x) \mathrm{d} x, \\
\mu(R) & =\int_{0}^{\infty} w(x) v^{2}\left(R x^{-1}\right) \mathrm{d} x,
\end{aligned}
$$

where $\mu(R) \equiv 2 \pi G R \Sigma(R)$. The integral kernel $w(x)$ involves the complete elliptic integrals of the first and second kind, $K$ and $E^{4}$, and is singular at $x=1$

$$
w(x)=\frac{1}{\pi}\left(\frac{K[k(x)]}{1+x}+\frac{E[k(x)]}{1-x}\right), \quad k(x)=\frac{2 \sqrt{x}}{1+x} .
$$

Accordingly, the integration in Eq. (1) and Eq. (2) should be understood in the Cauchy principal value sense. Given a $v(R)$, the $\Sigma(R)$ could be calculated directly from Eq. (2).

$$
{ }^{4} K(k)=\int_{0}^{\pi / 2} \frac{\mathrm{d} \varphi}{\sqrt{1-k^{2} \sin ^{2} \varphi}}, \quad E(k)=\int_{0}^{\pi / 2} \mathrm{~d} \varphi \sqrt{1-k^{2} \sin ^{2} \varphi} .
$$


In the literature, there are known integral expressions equivalent to Eq. (2) on an infinite domain $0<x<\infty$, for example [13], but they differ from each other by surface terms when cut off at a finite radius (this happens for real rotation curves measured only out to finite radii). Associated with this is the property of disk model, that the mass density in the region below the cutoff radius is affected by the assumed continuation of the rotation curve beyond the cutoff radius (see [17] for a more detailed discussion). The nonuniqueness in the mass density cannot be overcome without additional physical premises. The cutoff problem can be reduced when some data complementary to the rotation curve are available. At the galactic outskirts, where the density of stars is low enough, $\Sigma_{\text {gas }}$ is the main contribution to the total disk density $\Sigma$. One can approximate the amount of gas by $\Sigma_{\text {gas }}=(4 / 3) \Sigma_{\mathrm{H}}$, with $\Sigma_{\mathrm{H}}(R)$ being the hydrogen column density and the factor $4 / 3$ is introduced to take the helium abundance into account. Then, the iterative scheme proposed by Jałocha et al. [19] (or any equivalent) can be used. Based on the rotation curve and $\Sigma_{\text {gas }}$ observations, one is led, through repetitive use of integral transforms Eq. (1) and Eq. (2), to a global surface density profile (that continuously overlaps with $\Sigma_{\text {gas }}$ in the outer regions) and a global velocity profile (consistent with the rotation curve out to the cutoff radius).

In the subsequent section, we assume that the global profiles $\Sigma_{0}(R)$ and $v_{0}(R)$ have been already derived with the help of any method analogous to that in [19]. Then, we show how to modify the $\Sigma_{0}(R)$ further so that the isotropic velocity dispersion $\sigma(R)$ could be accounted for.

\subsection{Nonvanishing velocity dispersion}

For (axi-symmetric) circular streaming motion in the $z=0$ plane with the dispersion tensor isotropic in that plane $\left(\overline{v_{\phi}^{2}}-v_{\mathrm{c}}^{2}=\sigma^{2}=\overline{v_{R}^{2}}\right)$, the radial part of Jeans equations reduces to

$$
R^{-1} v_{\mathrm{c}}^{2}=\Psi^{\prime}+\Sigma^{-1}\left(\Sigma \sigma^{2}\right)^{\prime} .
$$

For such a motion, the azimuthal component of Jeans equations and the continuity equation are identically satisfied. For $z \neq 0$, we assume reflection symmetry $z \rightarrow-z$. By assuming the bulk density to be of a step function form: $\Sigma(R) / H$ for $|z|<H / 2$ and 0 for $|z|>H / 2$, and integrating the $z$-component of the second moment Jeans equation in the direction normal to the disk plane $\left(\partial_{z} \Psi=2 \pi G \Sigma\right.$ for an infinitesimally thin disk), we would end up with $\sigma_{z}^{2}=2 \pi G H \Sigma$ as an estimation of the vertical pressure needed to support a thin and finite width disk against its gravitational compression. Knowing $\sigma_{z}^{2}$ and $H$ from measurements, the formula would allow one to infer the column mass density $\Sigma$ (up to a geometric factor of order unity, 
depending on the vertical structure of the disk). Our study differs in this point from the usual approach: we will obtain the disk mass density $\Sigma(R)$ in a way independent of the disk's vertical structure.

We find it convenient to split $v_{\mathrm{c}}^{2}$ into a gravitational contribution $v_{\Psi}^{2} \equiv$ $R \Psi^{\prime}$, where

$$
v_{\Psi}^{2}=2 \pi G R \int_{0}^{\infty} \Sigma(R x) x w(x) \mathrm{d} x,
$$

and a contribution from the velocity dispersion term $v_{\sigma}^{2}$

$$
v_{\sigma}^{2} \equiv R \Sigma^{-1}\left(\Sigma \sigma^{2}\right)^{\prime}
$$

(the symbol $v_{\sigma}^{2}$ is formal, it can be also negative). In accord with Eq. (3), our task is to find a $\Sigma(R)$ which for a given $\sigma^{2}(R)$ gives a circular velocity profile

$$
v_{\mathrm{c}}(R) \equiv \sqrt{v_{\Psi}^{2}(R)+v_{\sigma}^{2}(R)}
$$

overlapping with the rotation curve. Below, we present an iterative scheme realizing this idea.

\subsubsection{The iterative procedure}

We assume that $\sigma(R)=\sigma_{0} f(R)$ with fixed function $f(R)$ and variable parameter $\sigma_{0}$. The iterations begin with a small $\sigma_{0}$, and a disk mass density $\Sigma_{0}(R)$ that precisely accounts for the observed rotation curve for $\sigma_{0}=0$. Each iteration step consists of three stages: $(i) v_{\sigma(1)}^{2}$ is calculated from Eq. (5) with $\Sigma_{0}$ substituted for $\Sigma,(i i)$ the $v_{\sigma(1)}^{2}$ is inserted into the transform Eq. (2), which yields a correction to the density profile $\delta \Sigma_{1}$ and hence the corrected density $\Sigma_{1}(R)=\Sigma_{0}(R)-\delta \Sigma_{1}$, (iii) $v_{\sigma(2)}^{2}$ is calculated from Eq. (5) with $\Sigma_{1}$ substituted for $\Sigma$. The $v_{\sigma(2)}^{2}$ will differ from $v_{\sigma(1)}^{2}$, therefore, the next iteration cycle must be performed. Again, $v_{\sigma(2)}^{2}$ is inserted into Eq. (2) which results in a correction $\delta \Sigma_{2}$ and a second corrected density $\Sigma_{2}(R)=\Sigma_{0}(R)-\delta \Sigma_{2}$. By going on with repeating the steps $(i)-($ iii $)$ until the difference $v_{\sigma(k)}^{2}-v_{\sigma(k-1)}^{2}$ decreases to an acceptably small value, one obtains a $k^{\text {th }}$ corrected profile $\Sigma_{k}(R)=\Sigma_{0}(R)-\delta \Sigma_{k}$. Now, by applying the integral transform Eq. (1) separately to all three terms in the equation $\Sigma_{k}(R)=\Sigma_{0}(R)-\delta \Sigma_{k}$, one has $v_{\Psi}^{2}=v_{0}^{2}-v_{\sigma}^{2}$ satisfied by construction. The iteration procedure can be repeated with a slightly different dispersion parameter, $\sigma_{0} \rightarrow \sigma_{0}+\delta \sigma_{0}$, and with the previous $\Sigma(R)$ now regarded as the first-step iteration density. This way, corresponding to a sequence of $\sigma_{0}$ values, a sequence of iterative solutions $\Sigma(R)$ is obtained. 


\section{Application of the iterative procedure to galaxy UGC 6446}

\subsection{The vanishing velocity dispersion (a comment on smoothing)}

Figure 1 shows a disk mass density $\Sigma_{0}(R)$ obtained for UGC 6446 by iterations, similarly as in [19], based on the rotation curve and the hydrogen density $\Sigma_{\mathrm{H}}(R)$ published by Verheijen and Sancisi [21]. We assumed that $\Sigma_{\text {gas }}(R)=\frac{4}{3} \Sigma_{\mathrm{H}}(R)$. In the same figure, the $\Sigma_{0}(R)$ is compared with the luminosity profile. It is seen that the corresponding local mass-to-light ratio would grow rapidly at $R \approx 12 \mathrm{kpc}$. Considering the high abundance of hydrogen, this growth could be explained by introducing a small fraction of unseen baryonic matter [11]. But, taking the velocity dispersion into account modifies this picture.

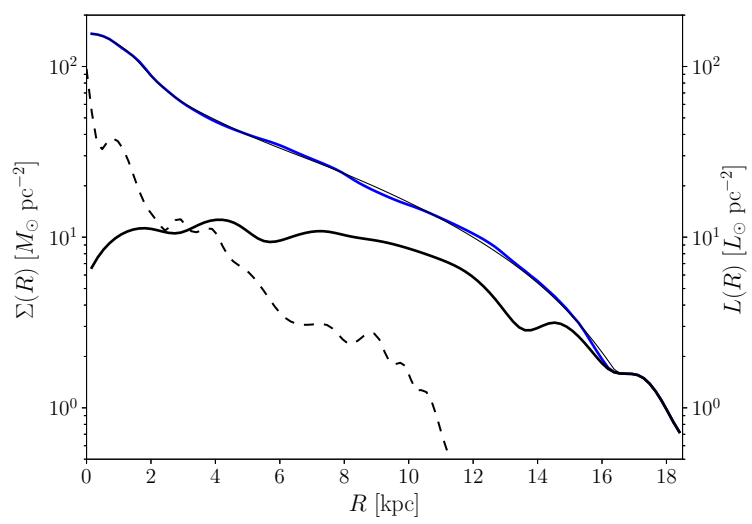

Fig. 1. The UGC 6446 disk mass density $\Sigma_{0}(R)$ derived from the rotation curve by the iterative method without the velocity dispersion. The thin solid line represents a smoothed cubic spline interpolation of $\Sigma_{0}(R)$, and it is compared with the usual cubic spline shown with the gray/blue line. The thick solid line is the gas column mass density $(4 / 3) \Sigma_{\mathrm{H}}(R)$. In the same plot, the Tully et al. [22] B-band luminosity profile is shown with the dashed line.

Below, we restrict ourselves to a simple model of the dispersion scalar $\sigma(R)$. This simplification neglects possible fluctuations in the dispersion due to the density fluctuations present at lower radii, however, the relative change in the density is small for lower radii on account of the density being high in that region. It is then reasonable to neglect small fluctuations in the density $\Sigma(R)$ by smoothing them out. Each time a $\Sigma(R)$ is found using Eq. (2) at a discrete number of points, we obtain a smooth $\Sigma(R)$ profile with the help of a smoothing cubic spline interpolation method. Although the difference between the smoothing cubic splines and the ordinary cubic splines is quite small, as seen in Fig. 1, this is not so for the derivatives $\Sigma^{\prime}(R)$. This is to be remembered when using Eq. (5). 
The rotation curve measurements are shown in Fig. 2. The circular velocity $v_{\mathrm{c}}(R)$ (solid line) was calculated with the help of the integral Eq. (1) for a smoothing cubic spline interpolation of $\Sigma_{0}(R)$. The smoothing affects the calculated rotation curve, nonetheless, the smoothed rotation curve agrees with the original rotation data well enough. For comparison, with the dotted line in Fig. 2, we show the best fit of the step profile $v(R)=V_{\mathrm{a}} \tanh \left(R / r_{\mathrm{s}}\right)$. A similar profile was used by the Disk Mass Survey [23] for a large number of galaxies. In Sect. 3.2.2, this will help us to estimate the order of magnitude of the stellar velocity dispersion expected for UGC 6446 .

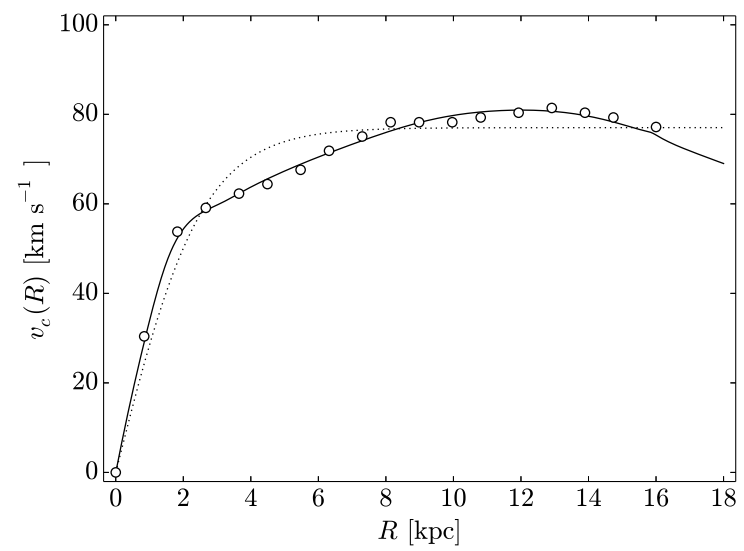

Fig. 2. The points represent the rotation curve of UGC 6446 determined by Verheijen and Sancisi [21]. The solid line is the rotation curve $v_{\mathrm{c}}(R)$ corresponding to the smoothing cubic spline of $\Sigma_{0}(R)$. The dotted line is the best fit of the step-like profile described in the text.

\subsection{The nonvanishing velocity dispersion}

To our knowledge, there are no measurements of the velocity dispersion available for UGC 6446 and we must rely on measurements available for other galaxies. Although the velocity dispersion observations are difficult, there is a hope more that such observations will be available in the nearest future. As for now, the Disk Mass Survey [24] provides data on the line-ofsight stellar velocity dispersion at particular galactocentric distances for a sample of 30 galaxies [23], while Tamburro et al. [25] give the gas velocity dispersion as a function of radius for selected 11 galaxies. Currently, there are precise methods of determining stellar column mass densities and the related stellar mass-to-light ratio profiles [26, 27].

In comparing the model predictions with the measurements, it should be remembered that the only direct observable is the velocity dispersion along the line of sight $\sigma_{\mathrm{LOS}}$. Splitting it into the normal and vertical components 
is model-dependent. For simplicity, we assume isotropic dispersion, that is, $\sigma=\sigma_{\mathrm{LOS}}$. This assumption may overestimate the $\sigma_{z}$ (e.g., Bershady et al. [28] find $\sigma_{z} / \sigma_{R}=0.6 \pm 0.15$ for the stellar component, however this estimation is based on many simplifying assumptions). Thanks to this choice, we avoid unnecessary complications and make the presentation of our method more transparent (if need be, the procedures can be generalized to more complicated forms of the dispersion tensor).

\subsubsection{Nonzero velocity dispersion for gas regions}

We start with by assuming that the velocity dispersion is nonzero only for the gas: $\sigma=\sigma_{\text {gas }}$. This is motivated by the fact that the rotation curve we use was obtained from the neutral hydrogen observations. The shape of the $\sigma(R)$ profile can be inferred based on those observed for other galaxies. The characteristic feature of this shape should be a significant drop-off at the stellar disk edge (where the $H_{\alpha}$ surface brightness drops off) followed by a linear decrease, as observed for galaxy NGC 6946 (Fig. 6 in Boomsma et al. [29]). Similar features are observed also in Tamburro et al. [25]: the dispersion profiles are decreasing functions of the distance with one or more step-like structures, often correlated with luminosity falloff, followed by a linear decrease from a value of about $10 \mathrm{~km} / \mathrm{s}$ down to about $5 \mathrm{~km} / \mathrm{s}$. These values are consistent with $\sigma_{\text {gas }}=11 \mathrm{~km} / \mathrm{s}$ adopted for the UGC 6446 by Watson et al. [4], which is the value characteristic of warm neutral medium.

We consider three model dispersion profiles $\sigma(R)$ (labeled as model A, $\mathrm{B}$ and $\mathrm{C}$, shown in Fig. 3) and study their influence on the local massto-light ratio in UGC 6446. These profiles are decreasing functions of the radius with a dropoff located at $R \approx 12 \mathrm{kpc}$ (where the observed brightness drops off significantly) followed by a linear decrease at the galaxy outskirts. The profiles differ between each other by their slopes at $R \approx 12 \mathrm{kpc}$. In applying the method of Sect. 2.2 to the three model profiles, one should put $\Sigma=\Sigma_{\text {gas }}$ in Eq. (5) in order to calculate $v_{\sigma}^{2}$. Because $\Sigma_{\text {gas }}$ is known from measurements, the $v_{\sigma}^{2}$ is fixed, and it is not necessary to repeat steps $(i)-$ (ii) in each iteration. This will change when we include the stellar velocity dispersion as then the $\Sigma(R)$ in Eq. (5) is altered in each iteration step.

By applying the iterative procedure, we obtain column mass densities $\Sigma(R)$ corresponding to the gas velocity dispersion profiles $\mathrm{A}, \mathrm{B}$ and $\mathrm{C}$ shown in Fig. 4. The resulting local mass-to-light ratio is shown in Fig. 5. As can be seen, in the region where the gradient of the velocity dispersion is large and the local mass density is low, the mass distribution is changed significantly. Then the local mass-to-light ratio could be reduced to values comparable to those in the inner part of the galaxy. 


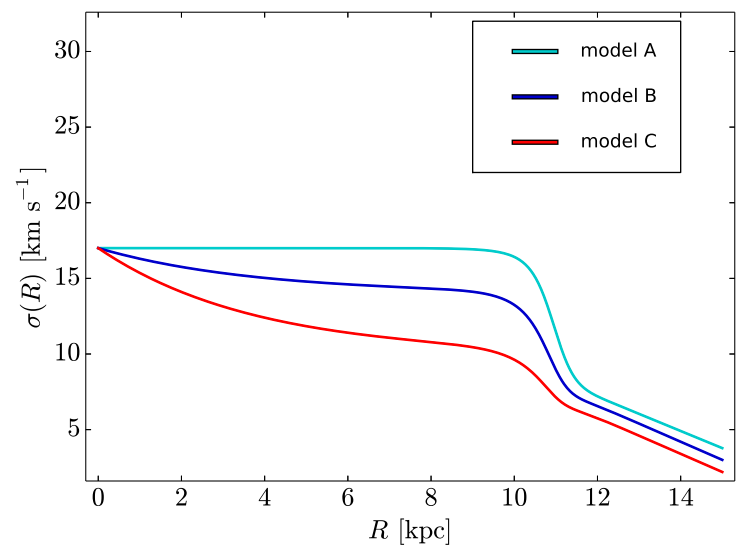

Fig. 3. The three toy models of the velocity dispersion profile for UGC 6446 described in detail in the text.

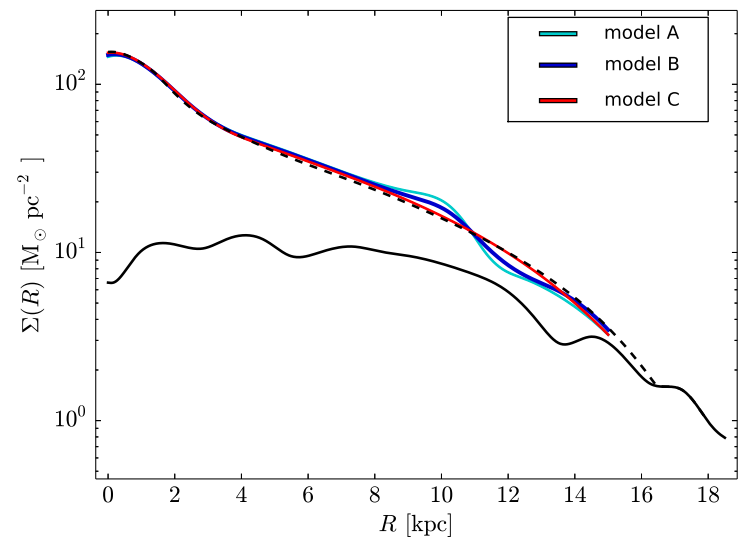

Fig. 4. The disk mass density $\Sigma(R)$ reconstructed iteratively for the gas velocity dispersion models $\mathrm{A}, \mathrm{B}$ and $\mathrm{C}$, respectively. The dashed line is the density distribution in the case without the velocity dispersion taken into account, and the thick black line is the gas density.

The presence of regions with substantial slopes in the dispersion profile is crucial. Such features are characteristic of the dispersion profiles in Tamburro et al. [25] and there may be more of them present in a particular galaxy, as it occurs for NGC 4736 and NGC 5055. Therefore, such a feature is likely to appear also for UGC 6446 close to $r=10 \mathrm{kpc}$, with the dispersion above $15 \mathrm{~km} / \mathrm{s}$. Although the proposed profiles are only toy models, they mimic the situation expected for UGC 6446. Of course, any doubts would be dispersed by a real measurement for UGC 6446 . 


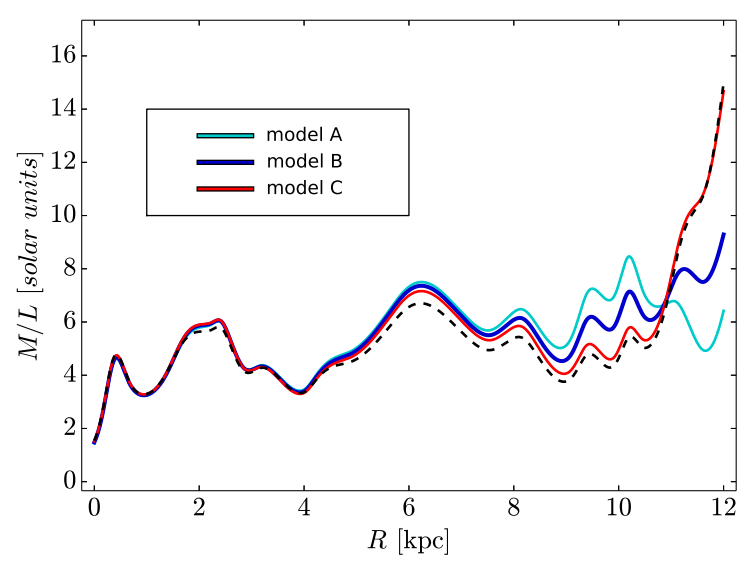

Fig. 5. The local mass-to-light ratio as a function of radius (we consider the B-band luminosity and the total gravitating mass after subtraction of the gas contribution), corresponding to the density distribution for models $\mathrm{A}, \mathrm{B}$ and $\mathrm{C}$, respectively. The dashed line is the local mass-to-light ratio for a mass density distribution without the velocity dispersion.

In the subsequent sections, we analyze to what extent this behavior could be changed in situations when also the stellar velocity dispersion is taken into account, and the velocity dispersion of gaseous regions was compared to the stellar velocity dispersion. In general, there could be separate rotation curves available, one for gas and another for stars, that would overlap with each other within some error limits. This assumption would be justified if the motion of matter was entirely driven by the gravitational interaction. In reality, there are other processes involved (like magnetic fields or SN explosions) that make the motion of gas regions different slightly from that of stars.

\subsubsection{The expected stellar velocity dispersion}

We should emphasized the importance of the Disk Mass Survey observations [23] with precise data on the stellar velocity dispersion $\sigma_{\mathrm{LOS}}$ as a function of radius for 30 galaxies from the UGC catalog. Although UGC 6446 is not included in their PPak sample, the expected velocity dispersion profile can be constrained based on these data. Martinsson et al. [23] model the projected rotation curves using a step-like profile

$$
v_{\mathrm{c}}(R) \sin i=V_{\text {arot }} \tanh \left(R / r_{\mathrm{s}}\right),
$$

with $V_{\text {arot }}$ and $r_{\mathrm{s}}$ as parameters. A de-projection $V_{\mathrm{a}}=V_{\text {arot }} / \sin i$ involves the inclination angle $i$, and assumes the stellar $\sigma_{\text {LOS }}$ to follow an exponential 
decrease law

$$
\sigma_{\mathrm{LOS}}(R)=\sigma_{\mathrm{LOS}, 0} \exp \left(-R / h_{\sigma, \mathrm{LOS}}\right) .
$$

In Fig. 6, we show the $\sigma_{\mathrm{LOS}, 0}$ and $V_{\mathrm{a}}$ parameters for 30 galaxies from the PPak sample (Fig. 2 shows the best fit we obtained for UGC 6446, with $V_{\mathrm{a}}=77 \mathrm{~km} / \mathrm{s}$ and $\left.r_{\mathrm{s}}=2.57 \mathrm{kpc}\right)$. A linear regression $\sigma_{\mathrm{LOS}, 0}=a V_{\mathrm{a}}+b$ to these data (with the best fit values $a=0.44$ and $b=-16.48 \mathrm{~km} / \mathrm{s}$ ) gives for UGC 6446 an estimate of the stellar velocity dispersion $\sigma_{\mathrm{LOS}, 0}=$ $17.4 \mathrm{~km} / \mathrm{s}$ at the galaxy center. This shows that the order of magnitude of the stellar velocity dispersion for this galaxy is comparable with the values of the velocity dispersion expected for the gas. Based on this premise, in the next section, we consider the situation in which the gas and the stars have the same velocity dispersion model profiles (shown in Fig. 3). This should be made with care, because the profile shapes could be slightly different. Nonetheless, the toy model profiles enable to fully use the iterative method presented in Sect. 2.2, and test to what extent the stellar velocity dispersion could change the results obtained in the previous section.

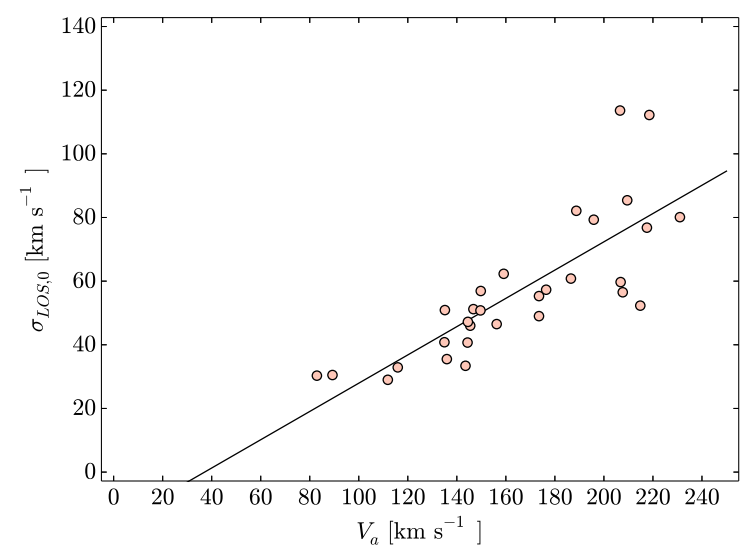

Fig. 6. The velocity dispersion parameter $\sigma_{\mathrm{LOS}, 0}$ and the de-projected rotation speed $V_{\mathrm{a}}$ for the PPak sample of the Disk Mass Survey. The solid line is a linear fit.

\subsubsection{The velocity dispersion of gas regions equated to that of stars}

Corresponding to a sequence of dispersion profiles $\sigma(R)=\sigma_{0} f(R)$, with a fixed function $f(R)$ for model A (defined by $\sigma_{0}=17 \mathrm{~km} / \mathrm{s}$ ) and free parameter $\sigma_{0}$, the iterations of Sect. 2 give in steps of $\delta \sigma=1 \mathrm{~km} / \mathrm{s}$ a sequence of corrected density profiles, as shown in Fig. 7 (for clarity, only selected $\sigma_{0}$ values are shown). As it is seen in Fig. 8, the density changes substantially at $R \approx 11 \mathrm{kpc}$, thus it will strongly influence the local mass-to-light ratio only in the neighborhood of the stellar disk edge. Figure 9 shows the local 


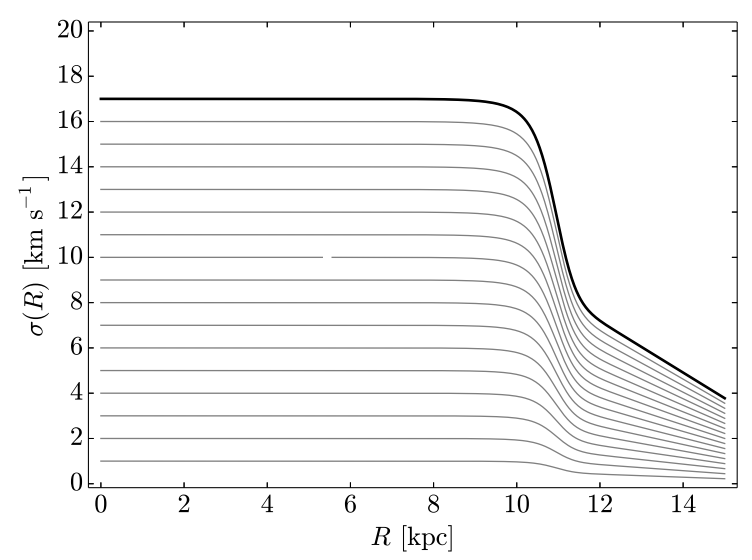

Fig. 7. A sequence of the velocity dispersion profiles $\sigma(R)=\sigma_{0} f(R)$ with various $\sigma_{0}$. The model A profile (black curve) has $\sigma_{0}=17 \mathrm{~km} / \mathrm{s}$.

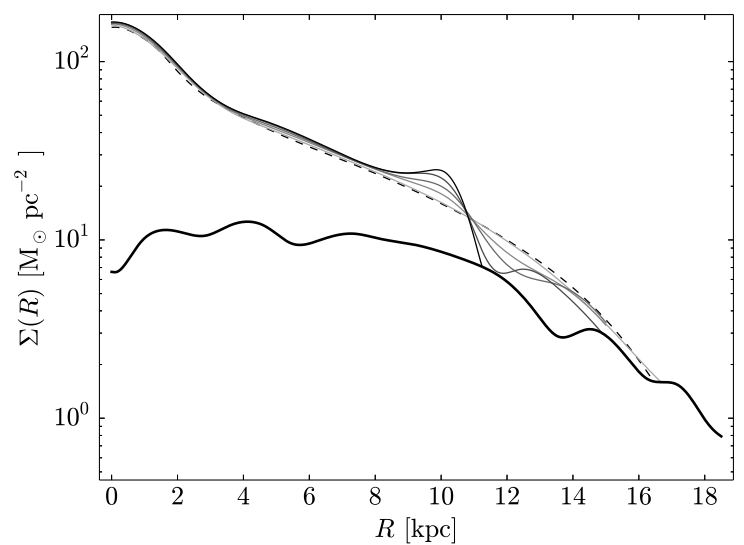

Fig. 8. Solid lines - a sequence of the disk density profiles $\Sigma(R)$ corresponding to the step-like velocity dispersion with parameters $\sigma_{0}=7,12,14,16,17 \mathrm{~km} / \mathrm{s}$, respectively. The profiles are shown using gray scale ranging from light gray for $\sigma_{0}=7 \mathrm{~km} / \mathrm{s}$ to dark gray for $\sigma_{0}=17 \mathrm{~km} / \mathrm{s}$. For comparison, with the dashed line is shown the density $\Sigma(R)$ without the velocity dispersion taken into account, and the black thick line represents the gas distribution.

mass-to-light ratio, obtained after subtraction of the gas contribution for the same sequence of $\Sigma(R)$ profiles. As follows from this figure, the behavior of the local mass-to-light ratio can be strongly modified at the stellar disk edge, from that rapidly increasing to decreasing. This proves that the inclusion of the velocity dispersion term in the disk model strongly influences the local mass-to-light ratio in regions with low mass density. 


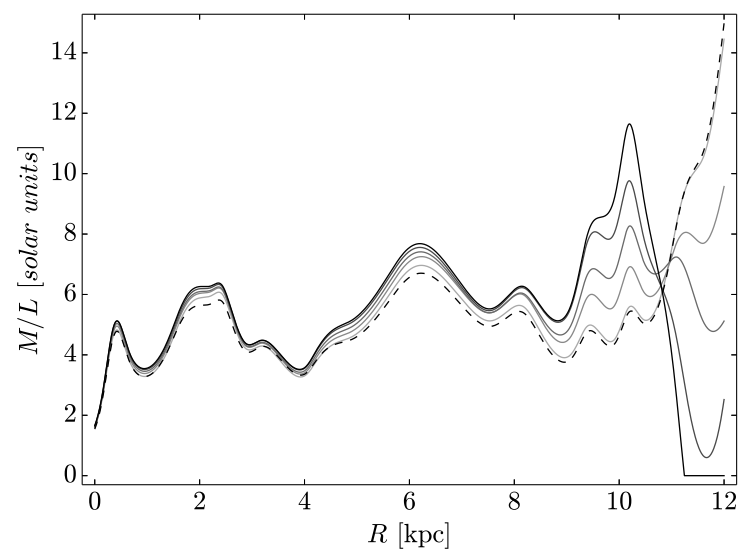

Fig. 9. The local mass-to-light ratio for the B-band luminosity and the total gravitating mass after subtraction of the gas contribution, shown for a sequence of density profiles $\Sigma(R)$ corresponding to a step-like velocity dispersion with parameters $\sigma_{0}=7,12,14,16,17 \mathrm{~km} / \mathrm{s}$, respectively. As in Fig. 8, these lines are represented on the gray scale, starting from light gray for $\sigma_{0}=7 \mathrm{~km} / \mathrm{s}$ to dark gray for $\sigma_{0}=17 \mathrm{~km} / \mathrm{s}$. The dashed line shows the local mass-to-light ratio for the case without velocity dispersion.

To convince ourselves that our procedure works well, we iteratively applied steps $(i)-($ iii $)$ to find the corrected density $\Sigma_{k}(R)$ for an example value of $\sigma_{0}=15 \mathrm{~km} / \mathrm{s}$. Figure 10 shows a sequence $v_{\sigma(k)}^{2}$ converging quickly after four iterations only. Although the difference $v_{\sigma(k)}^{2}-v_{\sigma(k-1)}^{2}$ appears not to

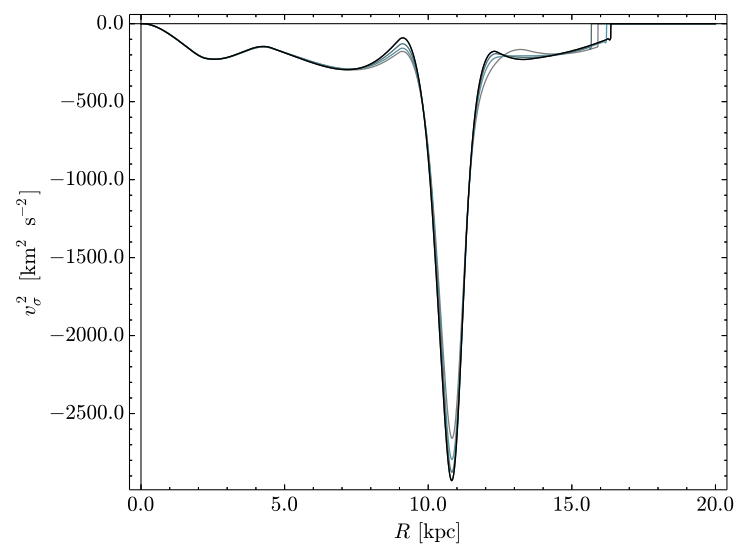

Fig. 10. A sequence of $v_{\sigma(k)}^{2}$ lines converging to the black line after four iterations steps $(i)-(i i i)$ described in Sect. 2.2. 
tend to zero for greater $k$ but to a small value (probably due to poor numerical resolution), the difference between ${\sqrt{v_{\Psi}(k)}}^{2}+v_{\sigma(k)}^{2}$ and $v_{\mathrm{c}}$ remains lower than $\approx 1 \mathrm{~km} / \mathrm{s}$, which is enough for our purposes. The $v_{\sigma}^{2}$ term is negative, which means that the gravitational contribution $v_{\Psi}$ is greater than the corresponding velocity on the rotation curve $v_{\mathrm{c}}$. This is intuitively clear as the action of the velocity dispersion term is similar in effect to a hydrodynamical pressure that must be balanced in the stationary case by increased gravitational force. In view of Eq. (1), the gravitational component $v_{\Psi}$ is the velocity for which the centrifugal force would equal the increased gravitational force. This is why we expect $v_{\Psi}>v_{\mathrm{c}}$ and this agrees with what is seen in Fig. 11. Contrary to the conclusion valid under spherical symmetry [12], we remind the remarks given earlier, that in disk model the increase in $v_{\Psi}$ does not necessarily imply higher abundance of mass.

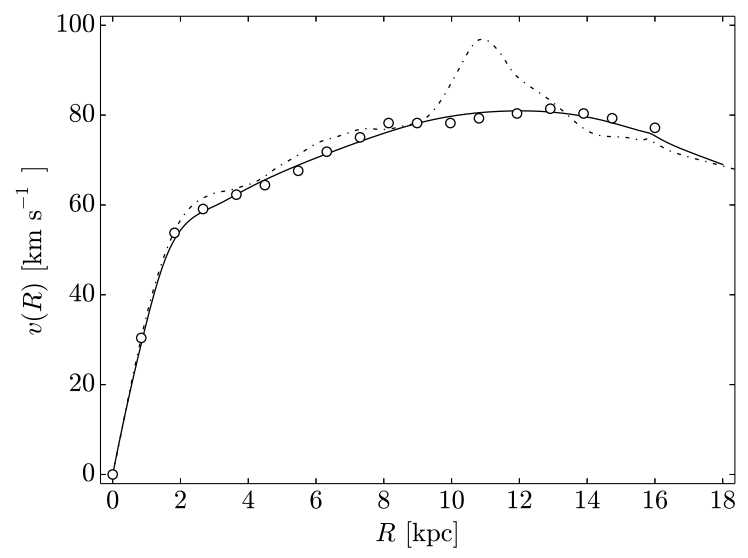

Fig. 11. The gravitational component of rotation, $v_{\Psi}(R)$, (dash-dotted line) calculated for the model A velocity dispersion profile with $\sigma_{0}=15 \mathrm{~km} / \mathrm{s}$, compared with the rotation curve (solid line) and the data points.

Below, we compare the model A with the results obtained for models $\mathrm{B}$ and C. In Fig. 12, we present the disk density distributions (obtained iteratively as described in Sect. 2.2) and in Fig. 13, we show the resulting local mass-to-light ratios, obtained after subtracting the gas contribution. It is evident from these figures that various kinds of the local mass-to-light ratio behavior are possible in the outer parts of the galaxy, depending on the velocity dispersion values in the stellar disk edge vicinity. The ratio may be left unchanged compared to that with no velocity dispersion (model $\mathrm{C}$ ), in which case the local mass-to-light ratio diverges at $R=12 \mathrm{kpc}$; it may converge to zero in the neighborhood of the stellar disk edge (model A); or remain oscillating around a value characteristic for the galaxy as a whole 
(model B). This proves that the velocity dispersion term may significantly modify the reconstructed values of the local mass-to-light ratio in the outer galactic regions.

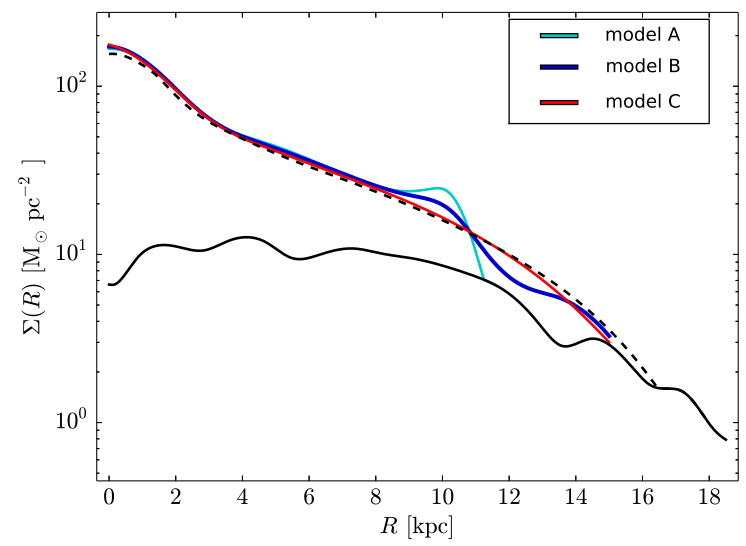

Fig. 12. The disk mass density $\Sigma(R)$ obtained iteratively for the velocity dispersion models A, B and C, respectively. The dashed line is the density distribution in the case without the velocity dispersion taken into account, and the thick black line is the gas density.

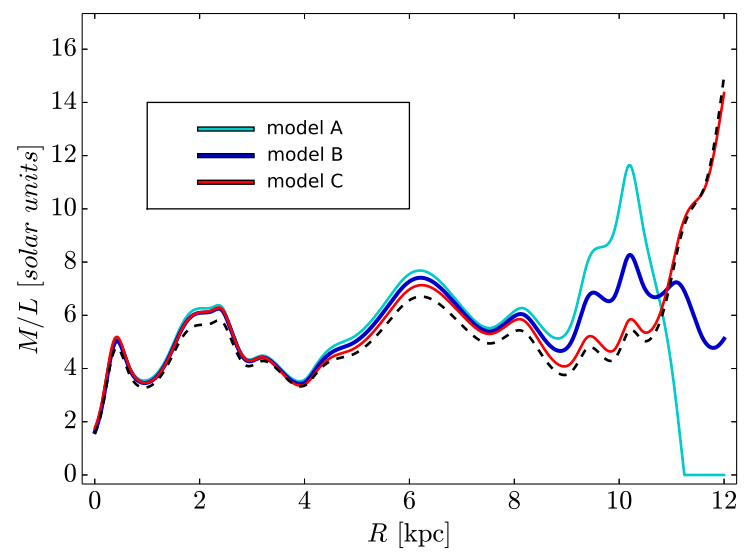

Fig. 13. The local mass-to-light ratio as a function of radius corresponding to the density distribution for models A, B and C, respectively (we consider the B-band luminosity and the total gravitating mass after subtraction of the gas contribution). The dashed line is the local mass-to-light ratio for a mass density distribution without the velocity dispersion. 
Figure 14 shows the gravitational part of the rotation curve $v_{\Psi}$ for the considered dispersion models. Similarly as for model A, the values attained by $v_{\Psi}$ are higher than the corresponding values attained by the rotation curve $v_{\mathrm{c}}$. The mass distribution in the disk has been modified in comparison with the situation without dispersion, such that the resulting correction to the gravitational force counterbalances the effective pressure due to the velocity dispersion term.

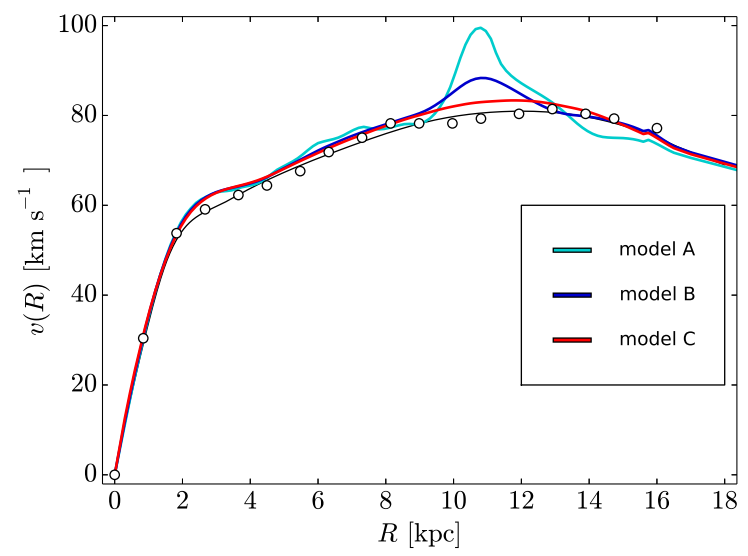

Fig. 14. The gravitational $v_{\Psi}$ velocity component obtained iteratively for the UGC 6446 galaxy with fixed model dispersion profiles A, B and C described in the text. For comparison, solid black line is the smoothed rotation curve $v_{\mathrm{c}}$ and the points show the rotation curve measurements.

3.3. The consequences of the velocity dispersion term for the mass function in the disk model

We would like to draw attention to the important fact that the expected increase in the gravitational potential due to the included dispersion term does not translate in the disk model into a noticeable increase in the mass function (or the total mass, in particular). This shows that the intuition about the role of the dispersion term in models with dominating spherical mass component, even those containing disks, does not apply for flattened galaxies - the increased gravitational potential due to the dispersion term does not mean accordingly increased mass function.

The mass functions corresponding to the surface densities of the considered models are shown in Fig. 15.

On comparing it with Fig. 11, it is seen that the relative increase of the mass function is much lower than the corresponding absolute value of the relative change in the gravitational term $v_{\Psi}$ (the relative change of the 
gravitational term in the disk model can be positive or negative). We stress this important result, because in models with dominating spherical mass distribution, the relative increase in the mass function would be everywhere exactly twice larger than the relative change in $v_{\Psi}$ (and the latter could not be negative).

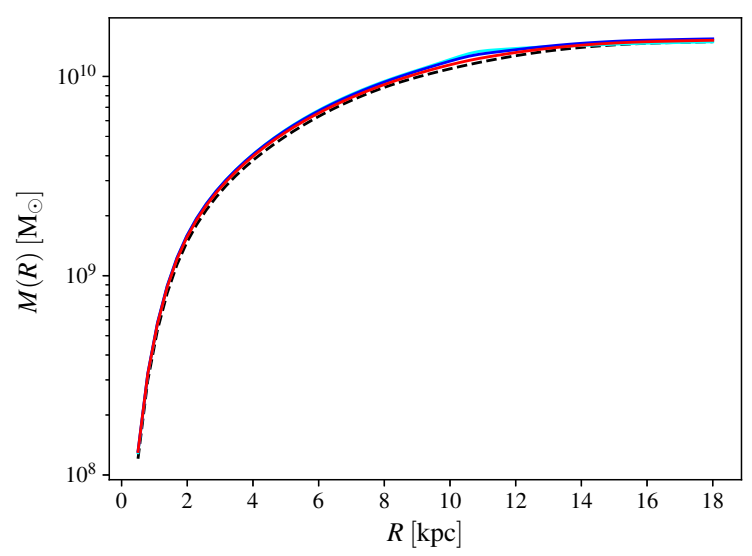

Fig. 15. The mass function for models $\mathrm{A}, \mathrm{B}$ and $\mathrm{C}$ corresponding to the surface mass densities from Fig. 12. The dashed line is the mass function in the case without the velocity dispersion.

\section{Summary}

\subsection{The model description}

In this work, we investigated the influence of the velocity dispersion term on the local mass-to-light ratio in the vicinity of the stellar disk edge in the framework of disk model of flattened galaxies, assuming a streaming motion about concentric circular orbits under axial symmetry and reflection symmetry with respect to the mid-plane, and that the mass distribution is flattened, that is, without a spherical mass component dominating at larger radii. The input quantities consist of the rotation curve $v_{\mathrm{c}}(R)$, the hydrogen column density $\Sigma_{\mathrm{H}}(R)$, and (isotropic in the disk plane, described by a single scalar $\sigma(R)$ ) a model of the velocity dispersion profile consistent with the measurements for a number of galaxies.

Our approach differs from other models in that we gave up the method of assumed shape disk density profile. This allows for precise fit into the measured rotation curve and for fully making use of the sensitivity of the disk model on the local structure in the mass density. Since the velocity dispersion contribution also involves the mass density, the relation between the circular velocity, the velocity dispersion and the mass density, becomes very 
intertwined. To overcome this difficulty, we found solutions by iterations. Furthermore, in our method, the local mass-to-light ratio is a variable output quantity, not a constant model parameter. These qualitative changes make our model more general and potentially useful in judging the importance of assumptions made by simpler models.

\subsection{The possible application}

The presented method can be applied to any spiral galaxy for which $v_{\mathrm{c}}(R), \Sigma_{\mathrm{H}}(R)$ and $\sigma(R)$ are known. The dynamical models of spiral galaxies usually ignore the role of the velocity dispersion in the surface mass density derivation. As we have shown on the example of galaxy UGC 6446, this simplification could be too restrictive when the outer parts of the galaxy are concerned, especially for flattened mass subcomponents. We chose the UGC 6446, because this galaxy is characterized by very high local mass-tolight ratio at the outskirts and, at the same time, there are features pointing to a disk-like distribution of baryonic matter with a possible small admixture of nonbaryonic dark matter. We already studied this galaxy in the past by neglecting the velocity dispersion, and found the above-mentioned peculiarity in the behavior of the local mass-to-light ratio at the boundary of the stellar disk. In this paper, we examined three model dispersion profiles and showed that in a region of low stellar mass density, the velocity dispersion may affect the resulting local mass-to-light ratio of this galaxy. Certainly, one cannot be sure if the influence of the dispersion is indeed responsible for the increase in the local mass-to-light ratio in UGC 6446. To establish this with certainty, a measurement of the velocity dispersion for this particular galaxy is indispensable, because the significance of the dispersion effect depends on the shape of the dispersion profile, its value, and where the increased slopes are located and how high they are. The availability of the precise velocity dispersion profile measurements is the main model limitation.

\subsection{Conclusions}

The most important result of our work is to notice that the velocity dispersion term in Jeans equations for flattened systems, may strongly influence the mass density profile in regions of low mass density. As a result of this, the local mass-to-light ratio at the galactic outskirts (where the density of matter is already low) can be substantially reduced without significant increase in the galaxy mass, which we illustrated on the example of galaxy UGC 6446. Along with galactic magnetic fields [30, 31], this is another factor which allows to reduce the local mass-to-light ratio, of which large values obtained in spherical models could be wrongly attributed to nonluminous matter. 
In the context of standard modeling of spiral galaxies, which usually splits the rotation curves into several subcomponents (the bulge, the disk and a dark matter halo), it is important to note that for nonvanishing velocity dispersion, the gravitational contribution to the rotation curve $v_{\Psi}$ of the flattened mass subcomponent may differ significantly from that curve, as it is evident from Fig. 14. Unlike in spherical symmetry, this difference may be explained merely by variations in the mass density, not necessarily implying an increase of the galaxy mass. This result strongly suggests that in addition to the standard components of the rotation curve, also the contribution from the velocity dispersion $v_{\sigma}$ should be taken into account. This effect may have consequences on the distribution of mass between various galactic components, in particular, in the disk and in the external halo.

\section{REFERENCES}

[1] Y. Sofue, Publ. Astron. Soc. Jpn. 69, R1 (2017).

[2] P.C. van der Kruit, L. Searle, Astron. Astrophys. 95, 105 (1981).

[3] A.K. Leroy et al., Astron. J. 136, 2782 (2008).

[4] L.C. Watson, Astrophys. J. 751, 123 (2012).

[5] T.P.K. Martinsson et al., Astron. Astrophys. 557, A131 (2013).

[6] A. Gallazzi, E.F. Bell, Astrophys. J. Suppl. Ser. 185, 253 (2009).

[7] V.C. Rubin, W.K. Ford, Jr., N. Thonnard, Astrophys. J. 238, 471 (1980).

[8] T. Takamiya, Y. Sofue, Astrophys. J. 534, 670 (2000).

[9] J. Schaye, Astrophys. J. 609, 667 (2004).

[10] P.C. van der Kruit, Astron. Astrophys. 466, 883 (2007).

[11] J. Jałocha, Ł. Bratek, M. Kutschera, P. Skindzier, Mon. Not. R. Astron. Soc. 406, 2805 (2010).

[12] J.J. Dalcanton, A.M. Stilp, Astrophys. J. 721, 547 (2010).

[13] A. Toomre, Astrophys. J. 138, 385 (1963).

[14] K.H. Nordsieck, Astrophys. J. 184, 719 (1973).

[15] A.J. Kalnajs, Rotation Curves of Galaxies in: Proc. of The Third Stromlo Symposium: The Galactic Halo, volume 165 of Astronomical Society of the Pacific Conference Series, (eds.) B.K. Gibson, R.S. Axelrod, M.E. Putman, 1999, p. 325.

[16] S. Casertano, Mon. Not. R. Astron. Soc. 203, 735 (1983).

[17] E. Bratek, J. Jałocha, M. Kutschera, Mon. Not. R. Astron. Soc. 391, 1373 (2008).

[18] P.D. Sackett, Publ. Astron. Soc. Australia 14, 11 (1997).

[19] J. Jałocha, Ł. Bratek, M. Kutschera, Astrophys. J. 679, 373 (2008). 
[20] Ł. Bratek, S. Sikora, J. Jałocha, M. Kutschera, Mon. Not. R. Astron. Soc. 451, 4018 (2015).

[21] M.A.W. Verheijen, R. Sancisi, Astron. Astrophys. 370, 765 (2001).

[22] R.B. Tully et al., Astron. J. 112, 2471 (1996).

[23] T.P.K. Martinsson et al., Astron. Astrophys. 557, A130 (2013).

[24] M.A. Bershady et al., Astrophys. J. 716, 198 (2010).

[25] D. Tamburro et al., Astron. J. 137, 4424 (2009).

[26] S. Zibetti, S. Charlot, H.W. Rix, Mon. Not. R. Astron. Soc. 400, 1181 (2009).

[27] E.E. Martínez-García, R.A. González-Lópezlira, G. Magris C., G. Bruzual A., Astrophys. J. 835, 93 (2017).

[28] M.A. Bershady et al., Astrophys. J. 716, 234 (2010).

[29] R. Boomsma, Astron. Astrophys. 490, 555 (2008).

[30] J. Jałocha, Ł. Bratek, J. Pękala, M. Kutschera, Mon. Not. R. Astron. Soc. 421, 2155 (2012).

[31] J. Jałocha, Ł. Bratek, J. Pękala, M. Kutschera, Mon. Not. R. Astron. Soc. 427, 393 (2012). 